\title{
In der Schule der Kritischen Theorie Die Frankfurter Seminare Theodor W. Adornos
}

Die Veröffentlichung von studentischen Protokollen akademischer Vorlesungen oder Seminare spielt in der Überlieferung philosophischer Lehren keine unerhebliche Rolle; viele der großen Vorlesungen Hegels sind uns nur aus den Mitschriften seiner Schüler bekannt, auch die legendäre Übung Martin Heideggers über Schillers »Briefe über die ästhetische Erziehung der Menschen « ist nur durch das Protokoll eines Studenten der Nachwelt überliefert. ${ }^{1}$ Gleichwohl mag man sich allein angesichts des Umfangs der hier anzuzeigenden Bände die Frage stellen, ob der Ertrag in diesem Fall den ungeheuren Aufwand tatsächlich lohnt; was im Folgenden zu lesen ist, sind alle noch aufzufindenden Protokolle der Seminare, die Theodor W. Adorno, häufig gemeinsam mit Max Horkheimer, nach der Rückkehr aus dem amerikanischen Exil in den Jahren von 1949 bis 1969 an der Frankfurter Universität gehalten hat. Zwei Bedenken sind es vor allem, die Zweifel an einem derart riesigen Unternehmen aufkommen lassen könnten. Erstens mag man sich fragen, ob es heute, nachdem aus dem umfangreichen Nachlass Adornos ein Dutzend seiner Vorlesungen bereits in vorzüglichen Editionen bei Suhrkamp vorliegen, tatsächlich sinnvoll ist, zusätzlich noch über zweitausend Seiten von Protokollen seiner Seminare zu veröffentlichen; reicht nicht die Lektüre der Vorlesungen vollkommen aus, so könnte der Einwand lauten, einen lebhaften Eindruck vom pädagogischen Engagement und von der intellektuellen Geistesgegenwart Adornos im akademischen Unterricht zu vermitteln? Schwerer noch dürfte aber ein zweites Bedenken wiegen, das nicht den spezifischen Wert der hier vorliegenden Seminarprotokolle, sondern das Genre solcher Dokumente insgesamt betrifft. Der Einwand liegt auf der Hand, studentische Protokolle akademischer Lehrveranstaltungen hingen viel zu stark von der Auffassungsgabe und dem Schreibvermögen des jeweiligen Verfassers oder der jeweiligen Verfasserin ab, um einschätzen zu können, ob der Verlauf einer Seminarsitzung angemessen und sinngenau wiedergegeben wird. Man mag dagegen einwenden, dass wir auch, wie erwähnt, unsere Kenntnis der Vorlesungen Hegels den Mitschriften seiner Schüler verdanken; aber das besagt wenig, hatte Hegel doch den kleinen Kreis seiner Studenten weitgehend selbst bestimmt und konnte er sich auf deren protokollarische Fähigkeiten mithin stark verlassen, während Adorno zu Beginn des Zeit-

1 Vgl. Martin Heidegger, Übungen für Anfänger. Schillers Briefe über die ästhetische Erziehung des Menschen. Wintersemester 1936/37. Seminar-Mitschrift von Wilhelm Hallwachs, hrsg. von Ulrich von Bülow, mit einem Essay von Odo Marquard, Marbach a.N. 2005 (Marbacher Bibliothek; 8). 
alters der Massenuniversität auf einen eher anonymen Kreis von Studierenden verwiesen war, denen das Protokoll anzuvertrauen häufig ein Risiko beinhalten musste. Insofern ist es sicherlich nicht falsch, zunächst einmal ein Fragezeichen hinter das Vorhaben zu setzen, auswahllos alle erhalten gebliebenen Protokolle der Seminarsitzungen Adornos aus der Nachkriegszeit zu publizieren.

Aber all diese berechtigt klingenden Zweifel werden schlagartig zerstreut, sobald man einmal mit der Lektüre der Protokolle begonnen hat, egal, wo man dabei einsetzt oder auf welches der Seminarthemen man sich stürzt. Was einen gleich zu Beginn für die vorliegenden Bände einnimmt, ist die durchgängig mustergültige Edition und Kommentierung der Seminarprotokolle. >Mustergültig، heißt in diesem Fall nicht nur, dass der Herausgeber Dirk Braunstein in Zusammenarbeit mit seinen sechs Mitarbeiterinnen und Mitarbeitern unendlich viel Mühe darauf verwendet hat, die Identität der jeweiligen Protokollantinnen und Protokollanten ausfindig zu machen, um deren Einwilligung zur Publikation einzuholen; wie viel Zeit, Tüftelei und Recherche es gekostet haben muss, die Spuren von den Namen der jeweiligen Autoren und Autorinnen bis hin zu ihren Adressen zurückzuverfolgen, kann man sich auch mit viel Phantasie wohl kaum vorstellen - und dass es auch immer wieder >Autorinnen ‘ waren, um deren $\mathrm{Zu}$ stimmung sich der Herausgeber kümmern musste, soll hier ausdrücklich betont werden, da es in den 1950er Jahren gewiss keine Selbstverständlichkeit für junge Frauen war, Philosophie oder Soziologie zu studieren. Mustergültig aber ist vor allem, wie die einzelnen Protokolle dann im Einzelnen mit Kommentaren und Verweisen versehen wurden; statt den großen, häufig gemachten Fehler zu begehen, möglichst viele Verweise auf die jüngste Forschungsliteratur zum gerade behandelten Thema einzustreuen und damit die Edition der Gefahr des schnellen Veraltens auszusetzen, werden in den Fußnoten resolut nur diejenigen Werke ausgiebig zitiert, die die Diskussionen oder die Stellungnahmen gerade behandeln. Durch diese kluge Entscheidung wird die Lektüre der Protokolle immer wieder - man glaubt es kaum - zum Vehikel philosophischer Erfahrungen, man vermag die Verwendung bestimmter Begriffe und Denkfiguren auf ihre Quelle zurückzuverfolgen, lernt den Stellenwert dieser begrifflichen Operationen im philosophischen Diskurs kennen und erhält obendrein als willkommene Zugabe noch die Stellungnahme Adornos $\mathrm{zu}$ all den verwickelten Gedankengängen. Wieder lässt sich kaum ausmalen, welche Anstrengung es den Herausgeber gekostet haben muss, für die meist nur kurz in den Protokollen herbeizitierten Ausdrücke und Argumente diejenigen Werkstellen ausfindig zu machen, auf die sich die oft unbelegten Aussagen mit ziemlicher Wahrscheinlichkeit beziehen; muss schon die Identifikation der Protokollanten und Protokollantinnen eine Sache geradezu detektivischer, wenngleich nicht immer erfolgreicher Bemühungen gewesen sein, so ist dieses Aufspüren der einschlägigen Quellen - sei es von 
Aristoteles, Leibniz, Durkheim oder Husserl - sicherlich das Resultat einer enorm zeitraubenden Suche in Büchern oder im Netz gewesen.

Trägt also schon die Edition dieses voluminösen Korpus in geglückter Weise zu einer spannenden, ja aufregenden Lektüre bei, so auch das beinah durchgängig hohe Niveau der abgedruckten Protokolle. Überlässt man sich erst einmal dem Lesen der durchschnittlich zwischen drei bis sieben Seiten langen Zusammenfassungen, so beginnt man sich unwillkürlich zu fragen, wie es hat möglich sein können, dass Adorno eine Schar so hochbegabter Studenten und Studentinnen um sich zu versammeln vermochte; die Protokolle, die er von seinen Seminaren verfassen ließ, sind fast immer stilistisch untadelig geschrieben, geben den Seminarverlauf meistens gut nachvollziehbar wieder und werden sehr häufig mit interessanten Kommentaren oder Rückfragen zum zuvor Wiedergegebenen versehen. Natürlich findet sich in der Masse auch immer mal wieder der ein oder andere Ausfall; da ist dann in der Zusammenfassung nur noch schwer auszumachen, worum die Seminardiskussion sich eigentlich drehte und wo die Frontlinien zwischen unterschiedlichen Auffassungen verliefen - interessanterweise handelt es ich dabei häufig um Texte, die den sprachlichen Duktus Adornos bis ins Letzte zu imitieren versuchen. Aber das Gros der Protokolle ist, wie gesagt, in der Qualität sowohl des Stils als auch des Gehalts außerordentlich eindrucksvoll. Ein Grund dafür mag sein, dass der Ruf Adornos als zeitkritischer Intellektueller und Denker während der 1950er Jahre schnell weit genug über Frankfurt hinausging, um einige der interessantesten jungen Köpfe aus der gesamten Bundesrepublik um seinen Lehrstuhl versammeln zu können; auf jeden Fall ist es auffällig, wie viele der Protokollantinnen und Protokollanten später bedeutende Intellektuelle oder Wissenschaftlerinnen bzw. Wissenschaftler werden sollten die Liste reicht von Peter Gorsen über Ivan Nagel, Regina Becker-Schmidt, Werner Mangold und Hans Friedrich Fulda bis zu Karl Markus Michel, wobei die ebenfalls gelegentlich protokollierenden Mitarbeiter und Mitarbeiterinnen Adornos und Horkheimers noch gar nicht genannt sind. Einige der hier abgedruckten Zusammenfassungen sind in sich so konzis und prägnant, dass sie damals ohne weiteres als Marginalien hätten veröffentlicht werden können.

Als ein eher zufällig gewähltes Beispiel sei auf das Protokoll verwiesen, welches ein leider nicht identifizierbarer Verfasser von der Sitzung vom 4. Juni 1957 des Hauptseminars »Zeitgenössische Ideologien • Begriff der Ideologie II» erstellt hat, das von Adorno gemeinsam mit Max Horkheimer veranstaltet wurde. Wenn auch nicht ganz auf dem hohen Niveau derjenigen Protokolle, die einige der zuvor namentlich Genannten verfasst haben, gelingt es dem Autor oder der Autorin erstaunlich gut, die wesentlichen Streitpunkte einer Diskussion wiederzugeben, die in jener Sitzung zwischen Karl Markus Michel und Adorno unter Mitwirkung von Horkheimer und Habermas geführt wurde. Der engagierte Disput 
entzündete sich an einem Referat von Michel, in dem dieser die These vertreten hatte, die bürgerliche Gesellschaft der Gegenwart bedürfe keiner neuen Ideologien zu ihrer eigenen Rechtfertigung mehr, weil sich die alten Illusionen von der segensreichen Wirkung edelmütiger und aufopferungswilliger Taten in Form von Klischees in der von allen konsumierten »mittleren Unterhaltungsliteratur« erhalten hätten - gemeint war alles von »Vicki Baum bis Norman Mailer «. ${ }^{2}$ Dagegen nun wandte sich Adorno in der Diskussion mit dem Einwand, Michel verfechte einen falschen Begriff der >Ideologie`, indem er darunter nur den zum geistigen Allgemeingut einer ganzen Bevölkerung herabgesunkenen Mythos des Bürgertums verstehe, jeder sei seines eigenen Glückes Schmied; was bei einer solchen zu simplen Vorstellung von >Ideologie` verlorenginge, sei der durchaus ernstzunehmende »Wahrheitsanspruch«, den alle Idealisierungen der gesellschaftlichen Realität in ihrer Entstehung erheben; insofern dürfe man heute, so Adorno, nicht von einem Verschwinden oder einer »Neutralisierung « aller Ideologien sprechen, sondern eher davon, dass es an einer reflexiven, wahrheitsorientierten Auseinandersetzung mit derartigen, häufig natürlich in Kitsch ausufernden, Idealisierungen der sozialen Realität inzwischen schmerzlich mangele. ${ }^{3}$ Wir können anschließend anhand des Protokolls auch noch nachvollziehen, mit welchem Argument sich Habermas auf die Seite von Michel schlägt und wie Horkheimer seinem Freund Adorno unter die Arme zu greifen versucht, indem er anrät, den Begriff der >Ideologie` nur den idealisierenden Vorstellungen des Bürgertums vorzubehalten.

Von derselben Dynamik und Spannung, die an diesem Protokoll einer Seminarsitzung sichtbar werden, sind die meisten der hier dokumentierten Lehrveranstaltungen Adornos geprägt. Man hat ständig den Eindruck, es ginge in den hitzigen Diskussionen nicht bloß um dieses oder jenes Element in der Geschichte der Philosophie und Soziologie, sondern stets um nicht weniger als `das Ganze`; kaum eines der Seminare, über deren Verlauf wir hier unterrichtet werden, läuft nicht an irgendeinem Punkt auf die Frage hinaus, wie es um die kapitalistische Gesellschaft heute in ihrer Totalität bestellt sei. Um diesen ungeheuren Anspruch nahezu aller der von Adorno bestrittenen Seminare noch einmal zu unterstreichen, soll kurz ein weiteres, wiederum eher zufällig ausgewähltes Protokoll herangezogen werden; es stammt aus der Hand des zuvor als Referent aufgetretenen Karl Markus Michel und ist der Sitzung vom 8. Juli 1954 des erneut mit Horkheimer veranstalteten Seminars zu Max Webers Wissenschaftstheorie gewidmet. Die Diskussion beginnt an diesem Tage eher zögerlich und kleinteilig, indem zu-

2 S. unten, S. 546.

3 S. unten, S. $545 \mathrm{f}$. 
nächst auf die Ambivalenz in Webers Begriff des >Idealtypus` Bezug genommen wird, der zwar nur »heuristisches Prinzip mit lediglich dienender Funktion beim Verstehen geschichtlicher Individuen« sei und doch auch einen Zug ins Normative aufweise, nämlich in die Konstruktion des geschichtlich "Seinsollenden « oder »Vorbildlichen «. ${ }^{4}$ Kaum ist dann aber der Hinweis gefallen, nach Webers eigener Überzeugung verlange eine solche normative Überschreitung der geschichtlichen Wirklichkeit regelmäßig den gekonnten Einsatz von »Phantasie«, nimmt die Diskussion im Seminar schnell an Fahrt auf und entlädt sich alsbald in weitausgreifenden Erörterungen - auf der Agenda steht plötzlich die Frage nach dem Schicksal der gesamten bürgerlichen Philosophie. Adorno wirft ein, nicht nur bei Weber, sondern auch bei anderen Denkern der Epoche - genannt werden Edmund Husserl und Max Scheler - finde sich die Tendenz, sich mittels der Phantasie in »Reaktion auf das Überwuchern des bloßen Daseins« in ein ideales Reich transzendierender Werte zu flüchten, ${ }^{5}$ Horkheimer macht anschließend geltend, Weber vermöge den »Positivismus « nur durch Import einer starken, theoriegeladenen Begrifflichkeit $\mathrm{zu} »$ retten «. ${ }^{6}$ Unschwer ist an beiden Interventionen zu sehen, dass es auch hier wieder um viel mehr geht als bloß die diskursive Überprüfung des Wahrheitsgehalts einzelner Theorien; was tatsächlich erneut zur Debatte steht, ist >das Ganze` der gegenwärtigen Welt mit ihren kulturellen Wucherungen und ihrer Verankerung in der ständig herbeizitierten kapitalistischen Realität.

Dieser Zug hin zu einem integralen Denken, das weder Disziplingrenzen noch methodische Zurückhaltung respektiert, mag ein weiterer Grund für die außerordentliche Attraktivität gewesen sein, die Adornos Seminare unter den damaligen Studierenden besessen haben müssen; viele von ihnen werden nicht nur deswegen in seine Lehrveranstaltungen gepilgert sein, weil ihm bereits ein Ruf als großer, öffentlich wirksamer Intellektueller vorauseilte, sondern weil es in jenen Veranstaltungen statt um die Vermittlung beliebiger Stoffe oder die bloße Aneignung klassischer Texte um die Fragwürdigkeit einer ganzen, nämlich der kapitalistischen Lebensform ging. Darin, diese geradezu existentielle Problematik in seinem pädagogischen Habitus zu verkörpern und sie noch im kleinsten Detail des besprochenen Stoffes erfahrbar zu machen, muss Adorno ein Meister gewesen sein; und wie durch Zauberhand scheint sich etwas von dieser Meisterschaft auf die besten der hier versammelten Protokolle übertragen zu haben.

Von hier aus fällt ein Licht auf den dritten Grund, warum die Leserin oder der Leser dieser Seminarprotokolle schnell von deren Lektüre gefesselt sein dürfte.

4 S. unten, S. 231.

5 S. unten, S. 232.

6 S. unten, S. $234 \mathrm{f}$. 
Schon aus den bislang veröffentlichten Vorlesungen Adornos wusste man, um wie vieles durchsichtiger und verständlicher seine Gedankengänge klingen, wenn sie nicht mehr in der Form seiner stilistisch durchkomponierten Schriften daherkommen; während in ihnen die Argumente fast ausnahmslos ohne Verweis auf die vielfältigen Erfahrungsbezüge präsentiert werden, aus denen sie letztlich stammen, geben die Vorlesungen diese ganz offenherzig und in großer Fülle zu erkennen - daher ihr stofflicher Reichtum, daher ihr ständiger Bezug auf prägende Lese-, Seh- und Höreindrücke, daher aber auch ihre ungleich leichtere Nachvollziehbarkeit. Generell zeigt sich in den Vorlesungen ein Adorno, der viel erfahrungsoffener, diskussionsfreudiger und irritierbarer ist, als man bei Kenntnis nur der Schriften hätte vermuten können. All das findet sich nun in den Seminarprotokollen nochmals gesteigert. Der in sich gekehrt wirkende, stilistisch jedes Wort abwägende und um sprachliche Strenge bemühte Denker tritt hier als ein ganz anderer auf, greift freimütig in jede Diskussion ein, indem er überraschende Verbindungen zur Erfahrungswelt der Studierenden herstellt, auf Parallelen eines theoretischen Arguments in Kompositionen, literarischen Texten oder Kunstwerken anspielt und sich überhaupt als ein ungemein quirliger, weltzugewandter und neugieriger Zeitgenosse erweist.

Bewunderungswürdig ist schon das breite Spektrum der Themen, die Adorno in seiner zwanzigjährigen Lehrtätigkeit in Frankfurt behandelte; es umfasst nicht nur die philosophischen Klassiker von Platon über Fichte und Kant bis zu Nietzsche und Bergson, nicht nur die soziologischen Theorien von Weber, Durkheim und Veblen, sondern schließt auch eine Reihe von gesellschaftstheoretischen Schlüsselproblemen ein, sei es nun die Frage nach einem angemessenen Begriff der Ideologie, nach der Idee des sozialen Fortschritts oder nach dem Konzept der sozialen Arbeitsteilung. Aber es ist natürlich nicht die Fülle der behandelten Stoffe als solche, die einen beim Lesen der Protokolle so schnell gefangen nimmt; ihre ungeheure Sogwirkung verdanken diese vielmehr den immer penibel festgehaltenen Momenten, in denen Adorno spontan das Wort ergreift, um aus der eigenen Erfahrung heraus zu erläutern, warum eine bestimmte These oder Formulierung besondere Aufmerksamkeit verdient. Das sind die Augenblicke, in denen man plötzlich der Kette von Assoziationen habhaft werden kann, die in seinem Denken ein einschneidendes Erlebnis mit einem abschlusshaft formulierten Gedanken verbindet - wie etwa, um ein kleines Beispiel zu nennen, aus dem Eindruck, den die Lektüre der Kriminalromane von Agatha Christie bei ihm hinterlassen hat, durch Generalisierung der Begriff der "Masche« entsteht, eine literarische Strategie der »Massenkultur«, die stets mit den Mitteln der 
»Pseudoindividualisierung « operiere. ${ }^{7}$ Von solchen Sekunden, in denen schlagartig der Motivationsgrund einzelner Thesen Adornos durchsichtig wird, berichten viele dieser Protokolle; sie lassen wunderbar erkennen, wie stark sein Denken sich der genauesten Registrierung von subjektiven Resonanzen verdankt, welche ein Buch, eine Komposition oder nur eine alltägliche Begegnung in ihm ausgelöst haben - in der »Negativen Dialektik» lautet die Formel für diesen Zusammenhang dann, dass dem Objekt umso eher ein Vorrang eingeräumt wird, je genauer ein möglichst sensibles, resonanzfähiges Subjekt dessen viele Eigenschaften wahrzunehmen vermag. ${ }^{8}$ An keiner anderen Stelle seines Schaffens legt Adorno ein beredteres Zeugnis von der damit behaupteten Dialektik ab als in den hier protokollierten Beiträgen zu seinen Seminaren.

Allerdings sind es in hohem Maße wieder die in den Anmerkungen angeführten Zitate, die es der Leserin oder dem Leser ermöglichen, bei der Lektüre den Weg vom individuellen Eindruck zum theoretisch sublimierten Gedanken nachzuvollziehen. Deutet Adorno in den Seminardiskussionen wieder einmal an, welche Kunst- oder Alltagserfahrung ihm als Anlass für einen seiner Gedanken oder Begriffe diente, so erfahren wir zumeist erst aus den präsentierten Belegen, wo sich die endgültige Formulierung der entsprechenden Schlussfolgerung in seinen Schriften findet. So erläutert Adorno etwa in einer Sitzung des Seminars zum Thema »Dialektik» im Wintersemester 1953/54, dass man sich den Realitätsgehalt des dialektischen Dreischritts aus These, Antithese und Synthese leicht an der Entwicklung des Kindes veranschaulichen könne; denn das heranwachsende Kind müsse zunächst an die »Allgüte« und die Ideale des Vaters sowie die »uneingeschränkte Liebe der Mutter« glauben, habe beides aber dann zwangsläufig in der Pubertät anhand der ihm von den Eltern beigebrachten Kriterien und Begrifflichkeiten zu negieren, bevor es schließlich als gereifter Erwachsener im gelungenen Fall beide Haltungen in Gestalt einer wachgehaltenen Erinnerung an das Glücksversprechen der Kindheit zur Synthese zu bringen vermöge. ${ }^{9}$ Dieser kurze Exkurs, protokoliert von dem späteren Soziologen Werner Mangold, spricht Bände, offenbart er doch in beinah rührender Form ein ganzes Bündel der Intuitionen, von denen das Denken Adornos zeitlebens zehrte: dass noch die abstraktesten Begriffe aus der reflexiven Verarbeitung von sinnlich Gegebenem, ja individuell Durchlebten stammen müssen; dass Kindheit fast immer das Versprechen eines >paradiesischen ` Aufgehobenseins und Geliebtwerdens beinhalte; dass bestimmte Negation die Triebkraft allen notwendigen Wandels sei und dass

7 S. unten, S. 545.

8 Vgl. GS, Bd. 6, S. 50 - 57.

9 S. unten, S. $159 \mathrm{f}$. 
schließlich ein gelungenes Dasein nur dort bestehen könne, wo das kindliche Sehnen und Glücksverlangen nicht an eine übermächtige Realität verraten wurde. Nun wird man sich jedoch schwertun, bündige Belege für diese Gedankenfolge im schriftlichen Werk von Adorno zu finden; nur da und dort blitzt einmal auf, dass für ihn ein enger Zusammenhang zwischen wiederangeeigneter Kindheit und dialektischem Schluss bestehen könnte. Die vorliegende Edition aber macht es den Suchenden leicht, indem sie in der Anmerkung einen Satz aus dem im Jahr des Seminars veröffentlichten Aufsatz »Valéry Proust Museum« wiedergibt, der aufs Schönste erhellt, welchen Niederschlag der zitierte Passus in den Schriften von Adorno gefunden hat. $^{10}$

Ein ganz anderes, aber ebenso eindrucksvolles Beispiel für die äußerst geglückte Weise, in der ein klar geschriebenes Protokoll durch geschickte Kommentierung so dargeboten wird, dass ein markanter Eindruck von der ingeniösen Präsenz Adornos im Unterricht entsteht, liefert die von Gerd Müller verfasste Zusammenfassung der in die Hochphase der Studentenbewegung fallenden Sitzung vom 14. Mai 1968 zu den Übungen zur Vorlesung »Einleitung in die Soziologie«. Auf dem Programm für die Sitzung stand ein Referat zur Kontoverse um die Wissenschaftstheorie Poppers, aber die Studierenden bestehen zu Beginn darauf, über den erforderlichen Widerstand gegen die geplante Notstandgesetzgebung zu diskutieren - über den Kontext der politischen Vorgänge, die zu diesem Vorschlag den Anlass geben, unterrichten die Anmerkungen in genau richtigem Umfang. Adorno, von den Seminarteilnehmerinnen und -teilnehmern genötigt, seine eigene Haltung $\mathrm{zu}$ dem Gesetzesvorhaben offenzulegen, bekundet in einer Mischung aus Trotz und Ironie, wer das nicht seinen Schriften entnehmen könne, der habe in einer soziologischen Übung nichts verloren. ${ }^{11}$ Die Auseinandersetzung über die vorgeschlagene >Umfunktionierung`der Veranstaltung zieht sich über mehrere Runden und mündet in Debatten über das Verhältnis von Theorie und

10 In Adornos Schrift Valéry Proust Museum [1953] heißt es über Paul Valéry: Museen adoriert er wie Gottes wahre Schöpfung, die ja, Prousts Metaphysik zufolge, nicht fertig ist, sondern kraft jeden konkreten Moments der Erfahrung, kraft jeder ursprünglichen künstlerischen Anschauung aufs neue sich ereignet. In seinem staunenden Blick hat er sich ein Stück Kindheit gerettet; ihm gegenüber spricht Valéry von Kunst wie ein Erwachsener. (GS, Bd. 10·1, S. 189) Den »Glücksanspruch aus der Kindheit« meldet Adorno auch in seiner Antwort Auf die Frage: Warum sind Sie zurückgekehrt [1962] an: Ich wollte einfach dorthin zurück, wo ich meine Kindheit hatte, am Ende aus dem Gefühl, daß, was man im Leben realisiert, wenig anderes ist als der Versuch, die Kindheit verwandelnd einzuholen. (GS, Bd. 20・1, S. 395)

11 Vgl. Die Frankfurter Seminare Theodor W. Adornos. Gesammelte Sitzungsprotokolle 19491969, hrsg. von Dirk Braunstein, Berlin und Boston 2021, Bd. 4, hrsg. von Dirk Braunstein, unter Mitw. von Nico Bobka, Maischa Gelhard, Jessica Lütgens, Hannes Weidmann, Lena Welling und Marcel Woznica, S. 517-524. 
politischer Praxis, bis Adorno die Reißleine zieht, um auf einer sofortigen Beschäftigung mit dem vorgesehenen Thema zu bestehen - und an der anschließenden, überaus anspruchsvollen Diskussion über die Wissenschaftslehre Poppers wird wunderbar deutlich, wie Adorno seine Kritik am Positivismus spontan mit Argumenten zu unterfüttern weiß, die in der vorangegangenen Kontoverse über das Verhältnis kritischer Theorie zum politischen Handeln bereits zentral waren. Wer wissen will, was und in welcher Weise philosophische Argumente zur reflexiven Erhellung und Durchdringung politischer Meinungsverschiedenheiten beitragen können, lernt es aus dem Protokoll dieser einen Sitzung im Mai 1968.

Ohne Mühe ließen sich immer neue Belege für die vielen Erkenntnisse unterbreiten, die diese Protokolle dank einer vorzüglichen Editionsarbeit und dank der großen Auffassungsgabe ihrer Autorinnen und Autoren in Hinblick auf die Genealogie der Ideenwelt Adornos liefern. Die bislang gegebenen Beispiele aber dürften bereits genügen, um davon einen lebendigen Eindruck zu vermitteln; nie zuvor, so lässt sich guten Gewissens behaupten, haben wir mehr über den Prozess in Erfahrung bringen können, in dem sich bei Adorno auf dem Resonanzboden sinnlicher Eindrücke und Erlebnisse allmählich die Gedanken formten, die seine Schriften präsentieren. Für die Forschung über seine Person und sein Schaffen wird diese Ausgabe der Seminarprotokolle daher in Zukunft eine wahre Fundgrube bilden; was sich darin an Einsichten über die Entstehungskontexte und Wirkungszusammenhänge der Werke Adornos, aber auch über die Stimmungslage und Fragestellungen der akademischen Jugend in den 1950er und 60er Jahren gewinnen lässt, dürfte an dokumentarischem Wert die Bedeutung so manch anderer Quelle aus jener Zeit weit überbieten. Es bleibt nur zu hoffen, dass diese Ausgabe trotz ihres abschreckenden Umfangs und trotz des zweifelhaften Rufs studentischer Protokolle ihre Leserinnen und Leser finden wird.

Axel Honneth 
